\title{
Carbon Nanotube-Metal Cluster Composites: A New Road to Chemical Sensors?
}

\author{
Q. Zhao, ${ }^{*, \dagger}$ M. Buongiorno Nardelli, ${ }^{\dagger, \ddagger}$ W. Lu, ${ }^{\dagger}$ and J. Bernholc ${ }^{\dagger, \ddagger}$ \\ Center for High Performance Simulation and Department of Physics, \\ North Carolina State University, Raleigh, North Carolina 27695-8202, and \\ CCS-CMS, Oak Ridge National Laboratory, Oak Ridge, Tennessee 37831-6359
}

Received January 26, 2005; Revised Manuscript Received March 15, 2005

\begin{abstract}
Novel carbon nanotube-metal cluster structures are proposed as prototype systems for molecular recognition at the nanoscale. Ab initio calculations show that already the bare nanotube cluster system displays some specificity because the adsorption of ammonia on a carbon nanotube-Al cluster system is easily detected electrically, while diborane adsorption does not provide an electrical signature. Since there are well-established procedures for attaching molecular receptors to metal clusters, these results provide a "proof-of-principle" for the development of novel, high-specificity molecular sensors.
\end{abstract}

Since their discovery in 1991, carbon nanotubes (CNTs) have become one of the most exciting nanomaterials, combining a range of extraordinary physical properties, such as extremely small size and high aspect ratio, high stiffness and excellent flexibility under different mechanical stimuli, high structural and chemical stability, and a rich spectrum of electrical properties. Many potential applications have been proposed: superstrong composites, energy storage and energy conversion devices, field emission displays, mechanical, chemical and biological probes and sensors, radiation sources, nanoelectronic devices, and more. ${ }^{1}$ In this letter we concentrate on the possibility of using carbon nanotubes as chemical sensors and probes. Pioneering experiments from Dai's group ${ }^{2,3}$ have shown that at room temperature the resistance of an individual single wall carbon nanotube is very responsive to the adsorption of molecules, such as $\mathrm{NO}_{2}$ and $\mathrm{NH}_{3}$. These measurements demonstrate the excellent sensing capabilities of CNTs, in particular their fast response time, high selectivity, and reversibility. It has also been shown that CNT-based single-molecule biosensors ${ }^{4-6}$ can compete with other nanowire nanosensors ${ }^{7}$ in detecting biological and chemical molecules. If appropriately functionalized, carbon nanotubes even have the ability to recognize proteins and DNA. ${ }^{8-10}$ Moreover, their intrinsic strength and resilience makes them ideally suited for ultrasmall sensors capable of exploring complicated geometries at the nanoscale. ${ }^{5,6}$ Real-world applications, such as CNT-

\footnotetext{
* Corresponding author. E-mail: zhao@nemo.physics.ncsu.edu.

North Carolina State University.

$\doteqdot$ Oak Ridge National Laboratory.
}

based resonant-circuit wireless ammonia sensors, have been developed by different groups. ${ }^{11,12}$

Due to their strong $\mathrm{sp}^{2}$ bonding and near perfect hexagonal network, carbon nanotubes are chemically stable and do not form strong chemical bonds with most molecules. However, since experiments have shown that the properties of a CNT can change when it is immersed in a specific chemical or biological environment, there has been a substantial effort toward the development of techniques to enhance the sensing capability of CNTs. The most common route to improvement in reactivity and sensitivity is through functionalization of CNT sidewalls with specific bio/chemical molecules. ${ }^{4-6,8}$ In fact, chemical functionalization can both ensure better chemical bonding between the nanotube and a specific chemical species as well as improve the selectivity of the adsorption process.

Alternatively, metal nanoparticles can be used to functionalize CNTs, enabling attachment of other species. Indeed, nanotubes coated with $\mathrm{Pd}$ nanoparticles become excellent $\mathrm{H}_{2}$ sensors. ${ }^{13}$ While $\mathrm{H}_{2}$ incorporates into bulk palladium so that the entire nanoparticle is an active part of the sensor, one could also consider sensors based on the reactivity of cluster surfaces. Since metal clusters have a broad range of diverse structures, they can provide a full range of reactivity with different molecules and can be easily functionalized for high selectivity by attaching specific receptors. The concept investigated in this letter is thus to use the carbon nanotube-metal cluster composite as the sensing unit of the device, where the cluster surfaces (or the receptors) act as the reactive sites for the adsorption of the foreign species. 


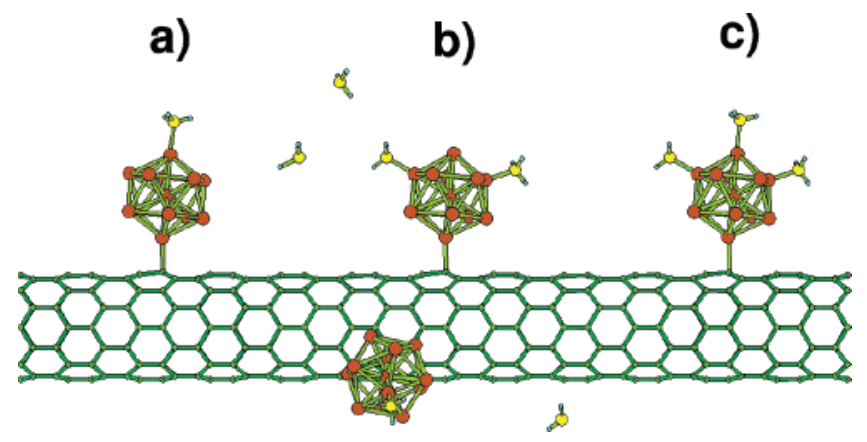

Figure 1. Illustration of carbon nanotube-metal cluster assembly for molecule sensing with metal clusters as the reactive sites. See text.

Monitoring the conductivity of this composite would then provide direct information about the chemical environment. In particular, the attachment of organic reagents and receptors to metal clusters has been the subject of many studies ${ }^{14}$ and their chemistry is well established. Methods for creating and dispersing metal clusters are also well known, and coating of nanotubes with clusters has already been achieved by electron beam evaporation, ${ }^{15}$ chemical attachment of preformed clusters, ${ }^{16}$ and precipitation from metal salt solution. ${ }^{17}$ However, to maximize the effect of the adsorbates on the cluster and on the nanotube-cluster interactions, the clusters should be relatively small. Figure 1 illustrates the concept of the nanotube/cluster sensor, where Al clusters and small molecules have been chosen as a paradigmatic example. We investigate this system theoretically below and show that it displays some initial specificity even without the use of receptors.

The calculations were carried out in the framework of density functional theory. We used the real-space multigrid method $^{18}$ in an $\mathrm{O}(\mathrm{N})$ formulation ${ }^{19}$ for the structural and electronic properties and an efficient Green's function method $^{20,21}$ for the evaluation of the quantum conductance. In this formulation, the Hamiltonian of the "conductor" system and of the infinite leads is expanded in localized orbitals. The effective size of the calculations is determined by the number of the localized orbitals. Since the quasiorder-N method uses variationally optimized orbitals adapted to the local environment, very few orbitals per atom are required for full convergence. The nearly linear scaling and relatively low computational cost of this method allow for accurate $a b$ initio treatment of the infinite leads in full atomistic detail and for a complete and consistent description of the coupling of the conductor with the leads.

Most of the calculations used local density approximation (LDA) for the exchange-correlation functional in the Perdew-Zunger parametrization of the Ceperley-Alder results ${ }^{22}$ and the Kleinman-Bylander ${ }^{23}$ procedure for incorporating nonlocal, norm-conserving pseudopotentials. As was well established in the past decade, LDA describes accurately the physics of carbon nanotubes and interactions with their environment, apart for a slight underestimation of bond lengths and an overestimation of formation energies. To confirm the validity of our conclusions, the most critical calculations were repeated using GGA-PBE. ${ }^{24}$ We found that
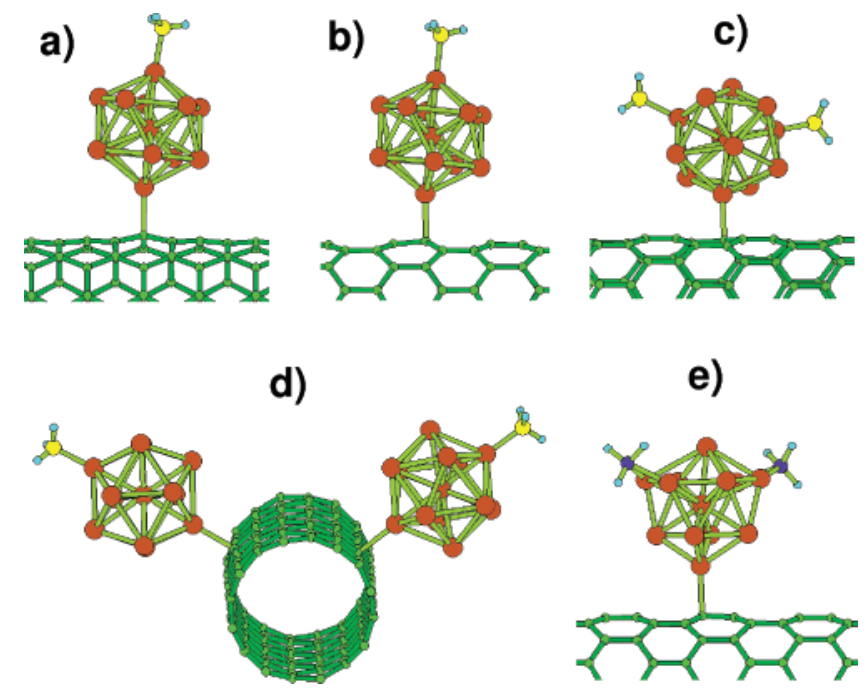

Figure 2. Typical atomic configurations of the $\mathrm{CNT}-\mathrm{Al}_{13}$ system with adsorbed molecules: (a) $(5,5)-\mathrm{Al}_{13}-\mathrm{NH}_{3}$, (b) $(8,0)-\mathrm{Al}_{13}-\mathrm{NH}_{3}$, (c) $(8,0)-\mathrm{Al}_{13}-\left(\mathrm{NH}_{3}\right)_{2}$, (d) $(8,0)-\left(\mathrm{Al}_{13}-\mathrm{NH}_{3}\right)_{2}$, and (e) $(8,0)-\mathrm{Al}_{13}-$ $\left(\mathrm{BH}_{3}\right)_{2}$.

the optimized structures, charge distributions, and quantum conductance spectra were well reproduced, with an average variation of $1 \%$. Binding energies showed larger variations (up to $10 \%$ ), but the qualitative characteristics of the bonding remained unchanged.

We have chosen to use an $\mathrm{Al}_{13}$-nanotube system as a paradigmatic example, since $\mathrm{Al}$ atoms are relatively easy to treat computationally. Although clusters of gold and other noble metals would be preferred for technological applications of nanotube-metal cluster sensors, the $\mathrm{Al}_{13}-\mathrm{CNT}$ system is sufficiently flexible to investigate the key concepts associated with nanotube cluster sensing. $\mathrm{Al}_{13}$ assumes an icosahedral structure ${ }^{25}$ and needs only one electron to close its electron shell with 40 electrons. Experimentally, $\mathrm{Al}_{13}{ }^{-}$ with 40 valence electrons exhibits the strongest shell closing effect and is very stable (it does not react with oxygen). Moreover, its derived binary clusters $\mathrm{Al}_{13} \mathrm{X}$ or $\mathrm{Al}_{12} \mathrm{X}(\mathrm{X}=$ $\mathrm{Cu}, \mathrm{Ag}, \mathrm{Au}, \mathrm{C}$, etc.) show enhanced stability and are potential building blocks for future metal cluster crystals. Our calculated binding energy of $\mathrm{Al}_{13}$ is $2.8 \mathrm{eV} /$ atom in GGA and $3.1 \mathrm{eV} /$ atom in LDA, which are in agreement with prior results. ${ }^{26}$

To determine the adsorption geometries of $\mathrm{CNT}-\mathrm{Al}_{13}$ assemblies before and after gas adsorption, we investigated several initial configurations. In each case, despite different starting distances and angles, the cluster ended up attaching to the nanotube in very similar "on top" configurations (see Figure 2), without encountering energy barriers. Similarly, the gas molecules adsorb onto the clusters without energy barriers. After atomic relaxation, both the nanotube and the $\mathrm{Al}_{13}$ cluster largely keep their original structures, while the bonding atoms are pulled slightly closer to each other. The binding energy of cluster adsorption, defined as $E_{\text {binding }}=$ $E_{\text {binding }}\left(\mathrm{CNT}+\mathrm{Al}_{13}\right)-E_{\text {binding }}(\mathrm{CNT})-E_{\text {binding }}\left(\mathrm{Al}_{13}\right)$, is 0.46 $\mathrm{eV}$ in the case of a metallic $(5,5)$ nanotube, with an equilibrium $\mathrm{C}-\mathrm{Al}$ bond length of $2.22 \AA$. A substantially stronger interaction characterizes the bonding between $\mathrm{Al}_{13}$ 

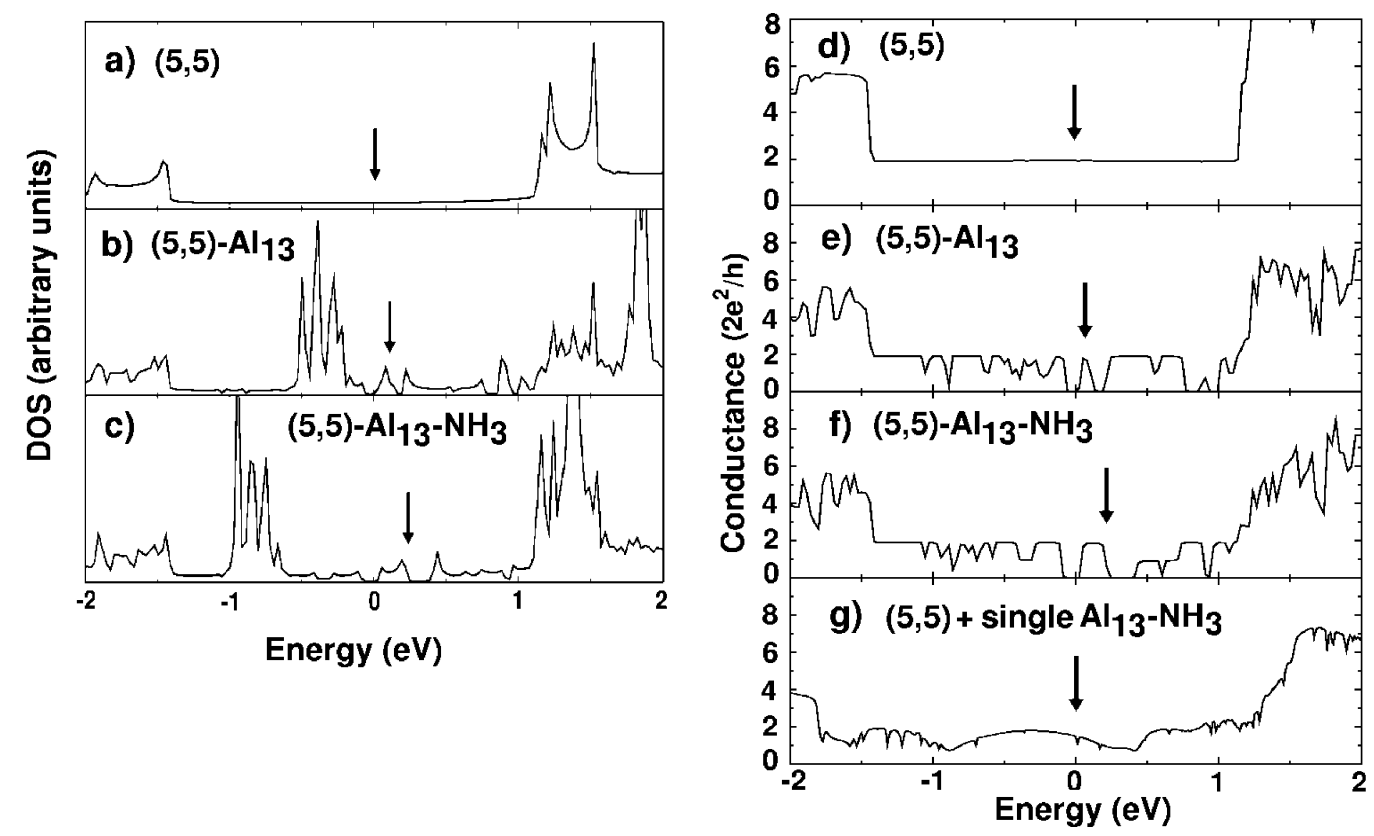

Figure 3. DOS and conductance of $(5,5) \mathrm{CNT}_{-} \mathrm{Al}_{13}$ assemblies. The energy zero is aligned with the Fermi energy of a clean $(5,5)$ tube, while the arrows mark the location of the Fermi energy in each panel. (a) DOS of a perfect $(5,5)$ tube, (b) $(5,5)-\mathrm{Al}_{13}$ assembly, and (c) $(5,5)-\mathrm{Al}_{13}-\mathrm{NH}_{3}$; (d-f) the respective conductances of the systems in (a)-(c). (g) Conductance of a $(5,5) \mathrm{CNT}$ with only a single $\mathrm{Al}_{13}-$ $\mathrm{NH}_{3}$ cluster attached. See text.

and a semiconducting $(8,0)$ nanotube of comparable diameter. This results in a shorter $\mathrm{C}-\mathrm{Al}$ bond, $2.15 \AA$, and a higher binding energy of $0.7 \mathrm{eV}$.

As mentioned earlier, the electrical conductance of carbon nanotubes is sensitive to molecular adsorption. The variations in the transport properties of the system are a measure of the sensitivity of nanotube-based chemical sensors. We have selected ammonia as a paradigmatic molecule for assessing the suitability of the $\mathrm{CNT}-\mathrm{Al}_{13}$ system. The choice of ammonia is due the fact that, as a well-known electron donor, it is one of the most widely used gases in experiments, and it is also important in industrial and medical applications.

The stable structure of the $\mathrm{CNT}-\mathrm{Al}_{13}$ system with a $\mathrm{NH}_{3}$ molecule adsorbed on the $\mathrm{Al}_{13}$ cluster is shown in Figure $2 \mathrm{a}-\mathrm{d} . \mathrm{NH}_{3}$ adsorbs preferentially on top of one of the vertexes of the cluster. For both the metallic $(5,5)$ and the semiconducting $(8,0)$ nanotubes, the adsorbed molecule enhances the bonding between the tube and the metal cluster without significantly altering their atomic geometries. Following the adsorption, the $\mathrm{C}-\mathrm{Al}$ bond shortens from 2.22 $\AA$ to $2.13 \AA$ for the $(5,5) / \mathrm{Al}_{13}$ assembly. The adsorption energy is $1.04 \mathrm{eV}$. For the semiconducting $(8,0) /$ cluster assembly, the adsorption energy is $1.1 \mathrm{eV}$ and the $\mathrm{C}-\mathrm{Al}$ bond length shortens from $2.15 \AA$ to $2.11 \AA$.

Since we are interested in the effects of the gas adsorption on the transport properties of the system, we need to characterize its electronic structure. In particular, charge transfer between the tube and the metal cluster provides important information regarding the system's electronic response. Not only does it affect the ionic component of the bonding but it also alters the position of Fermi level and the band alignment. In the clean $(5,5) \mathrm{CNT}-\mathrm{Al}_{13}$ assembly before $\mathrm{NH}_{3}$ adsorption, we calculate a charge transfer from the CNT to the $\mathrm{Al}_{13}$ cluster of about 0.1 electrons. ${ }^{27}$ This effect makes the nanotube effectively p-type, which is in agreement with previous experimental and theoretical results. Upon $\mathrm{NH}_{3}$ adsorption, the charge flows in the opposite direction and the CNT becomes electrically neutral. In fact, as an electron donor, $\mathrm{NH}_{3}$ donates about 0.2 electrons to $\mathrm{Al}_{13}$, which is redistributed in the system. The adsorption of $\mathrm{NH}_{3}$ also produces a substantial polarization and accumulation of charge in the region between the cluster and the nanotube. Moreover, the orbital hybridization between the nanotube and $\mathrm{Al}_{13}$ is enhanced, which is confirmed by the change in the formation energy and the bond length.

Turning to the electron transport properties, Figure 3 shows the conductance spectra and the densities of states (DOS) of a perfect $(5,5) \mathrm{CNT}$ and for a $\mathrm{CNT}-\mathrm{Al}_{13}$ system before and after $\mathrm{NH}_{3}$ adsorption. The perfect nanotube displays a quantized conductance of 2 (in units of $2 \mathrm{e}^{2} / \mathrm{h}$ ) due to the two crossing bands at the Fermi energy, a well-known result. The interaction of the clean tube with $\mathrm{Al}_{13}$ clusters deposited every 14 "carbon layers" modifies this idealized structure: the conductance is reduced significantly in some parts of the spectrum and especially near the Fermi level, where a small gap (about $0.1 \mathrm{eV}$ ) is created by the tube-cluster interaction. This gap is widened by about $0.2 \mathrm{eV}$ after $\mathrm{NH}_{3}$ adsorption, indicating that the interaction between the tube and the cluster becomes stronger. The $I-V$ curves calculated from these conductances are displayed in Figure 4a and show a dramatic decrease in the current after ammonia adsorption.

To further evaluate the consequences of molecular adsorption, we investigated the effects of increasing the number of adsorbed molecules per cluster. Adsorbing the second and the third $\mathrm{NH}_{3}$ molecules does not significantly change the $I / V$ characteristics (see Figure 4a), indicating that a relatively 

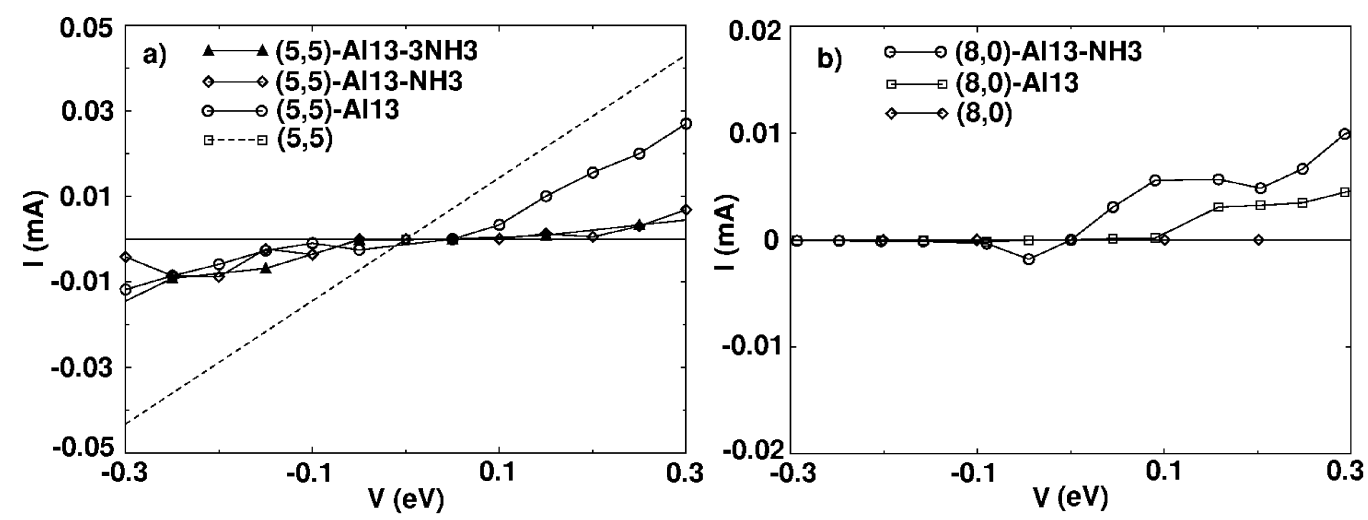

Figure 4. $I-V$ curves of $(5,5)-\mathrm{Al}_{13}$ assemblies (a) and $(8,0)-\mathrm{Al}_{13}$ assemblies (b).
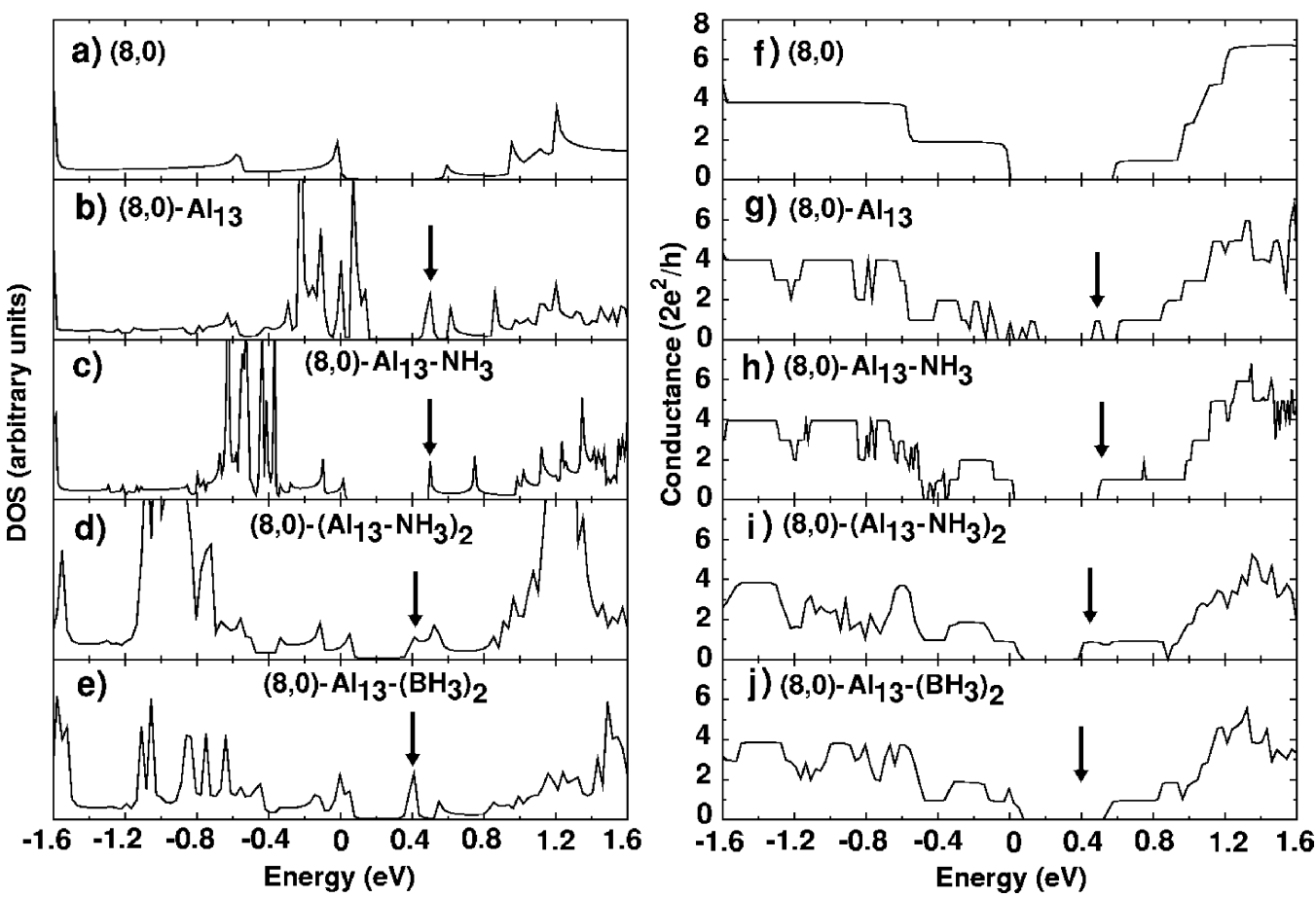

Figure 5. DOS and conductance of $(8,0)-\mathrm{Al}_{13}$ assemblies. The energy zero is aligned with the Fermi energy of a clean $(8,0)$ tube, while the arrows mark the location of the Fermi energy in each panel. (a) DOS of the perfect $(8,0)$ tube, (b) $(8,0)-\mathrm{Al}_{13},(\mathrm{c})(8,0)-\mathrm{Al}_{13}-\mathrm{NH}_{3},(\mathrm{~d})$ $(8,0)-\left(\mathrm{Al}_{13}-\mathrm{NH}_{3}\right)_{2}$, (e) $(8,0)-\mathrm{Al}_{13}-\left(\mathrm{BH}_{3}\right)_{2}$; ( $\left.\mathrm{f}-\mathrm{j}\right)$ are the conductances of the systems in $(\mathrm{a}-\mathrm{e})$, respectively. See text.

low concentration is sufficient to modify the electrical properties of the system.

Taking the opposite limit, we have also considered the case of a single metal cluster on a long carbon nanotube in a lead-conductor-lead geometry, using the techniques introduced in ref 21 . The conductance spectrum, which is shown in Figure 3g, looks very similar to that of a tube with a single topological defect. ${ }^{28}$ The conductance is reduced in most of the energy range, with the lowest value close to $1\left(2 \mathrm{e}^{2} / \mathrm{h}\right)$. In this situation, the interaction of the metal cluster with the nanotube introduces a "local defect" in the nanotube wall.

Since the semiconducting zigzag tube $(8,0)$ interacts more strongly with the $\mathrm{Al}_{13}$ cluster than does the $(5,5)$, the properties of the composite are influenced even more by the adsorption of ammonia. As in the $(5,5)$ tube, the charge transfer between the tube and the cluster in absence of $\mathrm{NH}_{3}$ occurs in the same direction and involves about the same fraction of electrons, leaving the tube p-type. However, after
$\mathrm{NH}_{3}$ adsorption the charge redistribution is quite different. In fact, $\mathrm{Al}_{13}$ draws more charge from $\mathrm{NH}_{3}$ (about 0.4 electrons) while, at the same time, the tube receives about 0.2 electrons from the metal cluster, changing its characteristics from p- to n-type. The $I-V$ curves in Figure 4a for the $(8,0)-\mathrm{Al}_{13}$ assembly clearly show the substantial increase in the conductivity due to gas adsorption.

The transport properties and the DOS of the $(8,0)-\mathrm{Al}_{13}$ system are summarized in Figure 5. The perfect $(8,0)$ tube is a semiconductor, with a gap of $0.6 \mathrm{eV}$ when computed in the local density approximation. The DOS of the $(8,0)-\mathrm{Al}_{13}$ system shows some localized $\mathrm{Al}_{13}$ states in the gap (including the HOMO of the system, which is now localized on the cluster). The Fermi level is pinned at the HOMO, which is energetically close to the conduction band. A significant change occurs after $\mathrm{NH}_{3}$ adsorption. Due to the density of clusters (one per $128 \mathrm{C}$ atoms), the charge transfer and orbital hybridization induced by adsorbing one $\mathrm{NH}_{3}$ per cluster led 
to merging of the bound state with the conduction band. The Fermi level is shifted there, with an estimated carrier density of about $10^{6}$ electrons per $\mathrm{cm}$.

We have also investigated the effect of an increased density of metal clusters on the system by computing the conductances and DOS for two $\mathrm{Al}_{13}$ clusters in the same supercell, while keeping them sufficiently far apart to avoid image and cluster-cluster interactions. As shown in Figure $5 i$, the energy gap decreases even more after ammonia adsorption and the conductivity of the system increases. The conductance also becomes more sensitive to the ammonia coverage and a more complex behavior is observed: if two ammonia molecules are adsorbed on a single $\mathrm{Al}_{13}$ cluster side by side, shown in Figure 1b, the system becomes a semiconductor again, while an additional ammonia molecule at the top of the cluster (Figure 1c) converts the system back to a conductor. This is due to the fact that there is no charge transfer in the configuration with two ammonia molecules, while in the three-molecule configuration the charge transfer is similar to that of a single molecule adsorption. The variation in charge transfer with different cluster coverages diminishes the sensitivity of this paradigmatic sensor configuration, but the electrical signature of $\mathrm{NH}_{3}$ adsorption remains detectable in a multicluster environment.

Finally, we investigated the selectivity of gas adsorption by also considering the $\mathrm{BH}_{3}$ radical and the $\mathrm{B}_{2} \mathrm{H}_{6}$ molecule. They are electron acceptors and behave quite differently than the electron-donating $\mathrm{NH}_{3}$. While $\mathrm{BH}_{3}$ is adsorbed on the top of the metal cluster without altering its atomic structure, just like the $\mathrm{NH}_{3}$ does, $\mathrm{B}_{2} \mathrm{H}_{6}$ dissociates upon adsorption and, unlike the two $\mathrm{NH}_{3}$, severely changes the atomic geometry of $\mathrm{Al}_{13}$. The two $\mathrm{BH}_{3}$ units, adsorbed on opposite corners of the icosahedron, "stretch" the cluster, (cf. Figure 2a and e), although the final $\mathrm{Al}_{13}-\left(\mathrm{BH}_{3}\right)_{2}$ structure still has high symmetry. While one $\mathrm{BH}_{3}$ does not change much the atomic and electronic structure of the tube-cluster assembly, the adsorption and dissociation of $\mathrm{B}_{2} \mathrm{H}_{6}$ loosens the bonding between the $(8,0)$ tube and the $\mathrm{Al}_{13}$ cluster, inhibiting charge transfer between the two. In both cases, however, the system remains insulating. In Figure $5 \mathrm{e}$ and $\mathrm{j}$, we show the DOS and the conductance of the $(8,0)-\mathrm{Al}_{13}-\left(\mathrm{BH}_{3}\right)_{2}$ assembly. As is clear from the figure, the adsorption of $\mathrm{B}_{2} \mathrm{H}_{6}$ introduces localized states in the gap region, but there are no extended states that can participate in conduction.

In conclusion, we have demonstrated that nanotube-metal cluster systems, represented here by the prototypical CNT$\mathrm{Al}_{13}$ composite, are indeed promising systems for the design of novel molecular sensors. Although the $\mathrm{CNT}-\mathrm{Al}_{13}$ assembly is not reusable for $\mathrm{NH}_{3}$ sensing because of the large adsorption energy, given the great flexibility in functionalization of metallic clusters, ${ }^{14}$ the CNT-metal cluster assemblies could be tailored for recognition of specific chemical agents with high selectivity and specificity. The key concepts are to use relatively small clusters and receptors that donate or accept a significant amount of charge upon adsorption of a target molecule, so that electron transport in the nanotube is affected. As demonstrated above, charge transfer of $\sim 0.1$ electrons per molecule is sufficient for detection. Although still at the "proof-of-principle" stage, we believe that CNT-metal cluster architectures provide a promising and important avenue for the development of nanoscale sensing devices.

Acknowledgment. This research was supported by DOE, NASA, ACS-PRF, and ONR. The calculations were carried out at DoD Supercomputing Centers.

\section{References}

(1) Baughman, R. H.; Zakhidov, A. A.; de Heer, W. A. Science 2002, 297, 787-792.

(2) Kong, J.; Franklin, N. R.; Zhou, C.; Chapline, M. G.; Peng, S.; Cho, K.; Dai, H. Science 2000, 287, 622-625.

(3) Zhou, C.; Kong, J.; Yenilmez, E.; Dai, H. Science 2000, 290, 15521555 .

(4) Besteman, K.; Lee, J.-O.; Wiertz, F. G. M.; Heering, H. A.; Dekker, C. Nano Lett. 2003, 3, 727-730.

(5) Erlanger, B. F.; Chen, B.-X.; Zhu, M.; Brus, L. Nano Lett. 2001, 1, 465-467.

(6) Wong, S. S.; Joselevich, E.; Woolley, A. T.; Cheung, C.; Lieber, C. M. Nature 1998, 394, 52-55.

(7) Cui, Y.; Wei, Q.; Park, H.; Lieber, C. M. Science 2001, 293, 12891292.

(8) Chen, R. J.; Zhang, Y.; Wang, D.; Dai, H. J. Am. Chem. Soc. 2001, $123,3838-3839$.

(9) Shim, M.; Shi Kam, N. W.; Chen, R. J.; Li, Y.; Dai, H. Nano Lett. 2002, 2, 285-288.

(10) Williams, K. A.; Veenhuizen, P. T. M.; de la Torre, B. G.; Eritja, R.; Dekker, C. Nature 2002, 420, 761-761.

(11) Ong, K. G.; Zeng, K.; Grimes. C. A. IEEE Sens. J. 2002, 2, 82-88.

(12) Chopra S.; Pham, A.; Gaillard, J.; Parker, A.; Rao, A. M. Appl. Phys. Lett. 2002, 80, 4632-4634.

(13) Kong, J.; Chapline, M. G.; Dai, H. Adv. Mater. 2001, 13, 13841386.

(14) See, e.g., Metal Nanoparticles: Synthesis, Characterization and Applications; Foss, C. A., Jr., Feldheim, D. L., Eds.; Marcel Dekker: New York, 2002.

(15) Zhang, Y.; Franklin, N. W.; Chen, R. J.; Dai, H. Chem. Phys. Lett. 2000, 331, 35-41.

(16) Jiang, K.; Eitan, A.; Schadler, L. S.; Ajayan, P. M.; Siegel, R. W.; Grobert, N.; Mayne, M.; Reyes-Reyes, M.; Terrones, H.; Terrones, M. Nano Lett. 2003, 3, 275-277.

(17) Choi, H. C.; Shim, M.; Bangsaruntip, S.; Dai, H. J. Am. Chem. Soc. 2002, 124, 9058-9059.

(18) Briggs, E. L.; Sullivan, D. J.; Bernholc, J. Phys. Rev. B 1995, 52, R5471-R5474. Briggs, E. L.; Sullivan, D. J.; Bernholc, J. Phys. Rev. $B$ 1996, 54, 14362-14375.

(19) Fattebert, J.-L.; Bernholc, J. Phys. Rev. B 2000, 62, 1713-1722.

(20) Buongiorno Nardelli, M. Phys. Rev. B 1999, 60, 7828-7833.

(21) Buongiorno Nardelli, M.; Fattebert, J.-L.; Bernholc, J. Phys. Rev. B 2001, 64, 245423-245427.

(22) Perdew, J. P.; Zunger, A. Phys. Rev. B 1981, 23, 5048-5079.

(23) Kleinman, L.; Bylander, D. M. Phys. Rev. Lett. 1982, 48, 14251428.

(24) Perdew, J. P.; Burke, K.; Ernzerhof, M. Phys. Rev. Lett. 1996, 77, 3865-3868.

(25) Yi, J.-Y.; Oh, D. J.; Bernholc, J. Phys. Rev. Lett. 1991, 66, 26332636.

(26) Rao, B. K.; Khanna, S. N.; Jena, P. Phys. Rev. B 2000, 62, 46664671

(27) Although there is no unique way of determining the amount of charge transfer between parts of a strongly bonded system, we were able to extract reliable values for the charge transfer by integrating the difference in charge densities between the whole system and its components, namely the cluster, the nanotube, and the molecule. The redistributed charge density is 1 to 2 orders of magnitude smaller than the total charge density, and the integrated values of the projection on the tube-cluster axis are not particularly sensitive to the point chosen to separate the tube and the cluster. The accuracy of estimating the transferred charge using this method is about 0.02 electrons.

(28) Buongiorno Nardelli, M.; Bernholc, J. Phys. Rev. B 1999, 60, R16338-R16341.

NL050167W 